\title{
Review on Developments in Fiber Optical Sensors and Applications
}

\author{
Venu Gopal Madhav Annamdas ${ }^{1,2}$ \\ ${ }^{1}$ School of Civil \& Environmental Engineering, Nanyang Technological University, 639798, Singapore \\ ${ }^{2}$ Laboratory of Monitoring Science, Online Research Platform, Hyderabad, 5000062, India
}

\begin{abstract}
The last couple of decades had witnessed a steep rise in extensive research of optoelectronic and fiber optical communication fields, which resulted in applications focused initially in military, aerospace equipments, and later in health monitoring for medicine, heritage culture and various engineering fields. Health monitoring has attracted the effort of many engineers throughout the world and is fast emerging as a pioneering field. It is helping to improve world economy in two ways; first, directly by providing longevity to all the important structures and secondly, by prevention of untimely failure leading to loss of life and money. This field is very vast, consisting of traditional monitoring methods as well as smart system based methods. The fiber optics belong to finest class of smart materials, there are many types and classifications of fiber optics based on necessity, manufacturer and the end user. This paper, gives a complete over view of fiber sensing systems and their usefulness. In addition, it highlights some of the general fiber optics available in the market with selected examples.
\end{abstract}

Keywords Optical Fibers, Sensors, Structural Health Monitoring, Damage Identification, Engineering, Heritage Culture, Health Care

\section{Introduction}

The last couple of decades have witnessed a steep rise in extensive research of optoelectronic and fiber optical communication fields, which resulted in applications focused initially in military and aerospace equipments, and later in health monitoring for medicine[1,2], heritage culture[3] and various engineering fields[4-7]. The recent optoelectronics industry has brought products such as compact disc players, laser printers, bar code scanners and laser pointers. The fiber optic communication industry has brought more reliable telecommunication links with higher performance and decreasing bandwidth cost. Especially these developments in both optoelectronics and fiber optical communication industries were used extensively in fiber optic sensor (FOS) technology. FOS technology in-turn has often been driven by the development and subsequent mass production of components to support these industries[8,9]. Mutual developments have resulted in optimization of components and prices with development of high quality products to replace traditional sensors for rotation, acceleration, electric and magnetic field measurement, temperature, pressure, corrosion, crack formation, acoustics,

* Corresponding author:

annamdas@ntu.edu.sg (Venu Gopal Madhav Annamdas)

Published online at http://journal.sapub.org/ijme

Copyright $(\underset{2}{ } 2011$ Scientific \& Academic Publishing. All Rights Reserved vibration, linear and angular position, strain, humidity, viscosity, chemical measurements and a host of other sensor applications. In general, an effective and reliable monitoring system should be supported by high performance sensory technology which fulfill 'A-to-E' characteristics as follows, (A)ccuracy: sensor should have a reliable accuracy; (B)enefit: commercial price of the sensor should be in a reasonable cost; (C)ompact: sensor should be small in size, especially for embedded installation; (D)urable: serviceability of the sensor should be long-lived; and (E)asy: sensor should be easy to operate and time consumed for retrieving data should be close to real time measurement[10]. Thus the FOS technology aptly fulfills such characteristics, which uses optical fiber either as the sensing element ("intrinsic sensors"), or as a means of relaying signals from a remote sensor to the electronic devices that process the signals ("extrinsic sensors"). A report on FOS[11] shows a phenomenal surge in United States market. It was valued at $\$ 235.0$ million in the year 2007. This is expected to reach $\$ 430.0$ million in 2009 and $\$ 1.6$ billion in 2014, for a compound annual growth rate (CAGR) of $30.1 \%$. Intrinsic sensors have the larger market share, worth $\$ 170.0$ million in 2007 and an estimated $\$ 306.0$ million in 2009. This is expected to reach $\$ 1.4$ billion in 2014, for a CAGR of $35.2 \%$. Extrinsic sensors generated $\$ 65.0$ million in 2007 and an estimated $\$ 124.0$ million in 2009. This segment is expected to reach $\$ 219.0$ million in 2014 for a CAGR of $12.1 \%$. Thus it is quite clear that the future of FOS industry is promising in USA. Even in countries like China or Russia 
and European Union, researchers are investigating and developing newer fibers, which are smaller than size of human hair. The overall market of FOS is quite large and it is expected to increase at a very steep growth rate.

\section{Fiber Optical Sensors}

\subsection{Flow of Electrons and Photons in Wires and Fibers}

Photons are electrically neutral as they need not carry any charge. They do not have mass at rest, and their mass is negligible in motion as compared to the electrons. In a wire, electrons require a "push" (voltage) to overcome inertial and electromagnetic forces. Voltage generates flow of electrons (current) and in-turn generates heat in the wire. Moreover, the flow of electrons creates magnetic fields that extend beyond the boundaries of the wire. These fields may influence electrons traveling in nearby wires to create currents in them. These wires can also act as unwanted antennas, where electromagnetic radiation passing by the wire can stimulate a flow of electrons. These effects can create "crosstalk" between wires, leading to degradation of sensors. Additionally, these metal wires can attract lightning strikes causing failure to the equipments[12] or the sensors may themselves under go changes in presence of external factors. However, photons do not have any of these conditions associated with implementation of FOS systems. They do not create electric or magnetic fields even in the same fiber, thus effectively eliminating crosstalk. There is no interaction between two adjacent photons travelling in the same fiber unlike two adjacent electrons travelling in a single wire. Thus two wires or group of wires cannot be connected to single wire to avoid repulsion of electrons due to their common electric charge. Whereas, it is possible to combine an extra-ordinarily large number of signals onto a single fiber, such arrangement is known as multiplexing. Since no electrical signal is carried on the fiber, it is safer in harsh environments like presence of explosive gases or fuels. In some applications, weight and volume may not be critical but for many other applications, weight and volume can be extremely important, and minimizing these can be very critical[13,14].

\subsection{Basic Fiber Composition}

Basically, a fiber is made of a glass or plastic that carries light throughout its length. Fiber sensors are sensitive to any parameter which can modify the intensity, frequency, polarization, or phase of light traveling through the fiber. The ability to interrogate numerous sensors multiplexed[15-18] along a single fiber permits an entire structure to be outfitted with sensors with a manageable number of leads routed to central access points. They facilitate transmission of light over longer distances and at higher bandwidths (data rates) than other forms of communications. Fibers are used instead of metal wires because signals travel along these fibers with less loss.
The basic fiber structure needed to guide the light wave consisting of a core surrounded by a cladding as shown in Fig. 1(a). Moreover, the light 'rays' should remain within the fiber, which is possible only if the 'index of refraction' of the core is always greater than the cladding i.e the core and cladding indexes need not be constant but core index must be always greater. However, the fiber with constant core index is simple to fabricate and is usually known as step index fiber.

Typical communicational-grade optical fiber sensors appear as long strands of glass material, with a core diameter of less than 100 microns. They usually have a core index of refraction between $1.450-1.480$ radians. Plastic fibers with core diameters of $2 \mathrm{~mm}$ are usually utilized for certain short distance applications $(<500$ meters $)$. In absence of any cladding, the core can be "immersed in air", which has an index of refraction of about 1.0 and the critical angle of incidence (greater the angle, effective is the fiber) would be close to the 41.8 degrees. Core and cladding diameters usually are specified together, for example, " $85 / 125$ " refers to a fiber that has a core and cladding diameters as 85 and 125 microns, respectively. However, the latest fiber structure for use in National Aeronautics and Space Administration (NASA) is of a size of human hair. Core and cladding diameters of these sensors[19] are too small as compared to sizes of previous decade fiber structure.

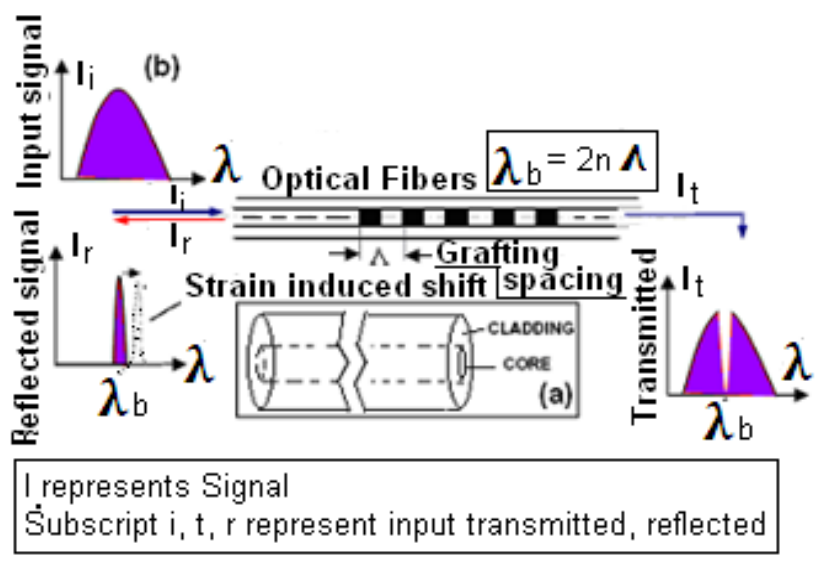

Figure 1. FOS and FBG sensors (a) FOS fiber composition (b) principle of FBG

\subsection{Important Features of Fiber Sensors}

The FOS consists of a thin optical fiber, typically ranging from 75 microns to 125 microns in diameter, can be configured to measure a wide range of effects via changes in light beams that are propagating through the optical fibers. The important features are (1) Immunity to electromagnetic interference: protects from lightning strikes and electrical hazards (2) Rugged and robust: can withstand harsh environment and temperature either on or inside any composite. (3) Multiplex: many fiber sensors are inter connectable to result in extremely vast sensing network along a single fiber line. (4) Multifunctional capabilities: 
multiple parameters can be sensed along the same fiber line simultaneously, such as strain, pressure, corrosion, temperature etc[20]. (5) Optimized size: fiber sensors influ- ence neither the stiffness nor disturb the integrity of the host material. (6) Optimization of cost: a high degree of synergy with the telecommunication and optoelectronic markets can make the prospect of low cost, high performance devices very likely in the future communicational[12]. (7) Control Systems for effective structural health monitoring (SHM): deployment of fiber sensor arrays to assess the condition of a structure. For example: FOS system deployed (to measure strain, temperature or environment) at important locations on any structure. Moreover the FOS system can also be used not only to collect the data but also to respond to it. For example: a highway that senses an accident and activates warning signs as a result of such deployment.

\subsection{Fabrication and Types of Optical Fibers}

Optical-grade silica-glass is the predominant material for communicational[12] and sensing applications. The glass used for optic fiber is many times clearer than normal window. We could construct a pane of glass several kilometers thick and still match the clarity of a normal window. If water were this clear we would be able to see the bottom of the deepest parts of the ocean[21]. A fiber optic system using a glass fiber is certainly capable of carrying light over long distances. By converting an input signal into short flashes of light, the optic fiber is able to carry complex information over distances of more than a hundred kilometers without additional amplification. This is at least five times better than the distances attainable using the best copper coaxial cables. The system is basically very simple: a signal is used to vary, or modulate, the light output of a suitable source - usually a laser or an light emitting diode (LED). The flashes of light travel along the fiber and, at the far end, are converted to an electrical signal by means of a photo-electric cell.

Silica-glass fibers are often doped with materials for instance Germanium dioxide and Aluminum dioxide to improve performance through changing the index of refraction and some other properties of the fiber. Hill et al[22] as the first to report photosensitivity in germanosilicate optical fibre and its application for fabrication of reflection filter. From there on, regularly never developments evolved in fiber technology. We occasionally use plastic for optic fiber but its losses are still impossibly high for long distance communications but for short links of a few tens of meters it is satisfactory and simple to use. In the recent years[23,24], plastic fibers with performance sufficient for short distance communications or cold light illuminating applications (typically less than 500 meters, and bandwidths less than $200 \mathrm{MHz}$ ) is increasing. It is finding increasing applications in hi-fi systems, and in automobile control circuitry. However plastic fibers are thicker than glass fibers by at least a millimeter, and can be handled without special tools or techniques. They were also signi- ficantly used due to their low cost and high fiber quality. For example, large-core plastic fibers (>500 microns) can illuminate sufficient light without significant heat, for applications in car-dash instruments, equipment-inspection light sources, medical instrumentation and image applications[25] with no-electrical requirements. Irrespective of whether FOSs are silicon or plastic based, their applications are in telecommunications, ships, submarines, satellites, office buildings, trains, automobiles, manufacturing plants, scientific labs, trans-oceanic communications links, aerospace, oil and pipeline, civil infrastructures $[6,17$, $26,27]$, biomedical[28], healthcare systems [1,29] and everywhere communication or data is exchanged[12][See later sections for details].

FOSs can be classified into four main categories based on intensity, phase, wavelength and polarization modulations[30,31]. However, these four categories can be treated as one category which is simply based on modulation or dem- odulation to measure shifts in amplitude, phase, frequency etc. Another classification is based on application type such as physical, chemical or biomedical sensors. Whether classification is based on modulation or application type, there organization either in the form of continuously dist- ributed systems or the quazi-distributed systems is describe- ed mostly. Usually, when a structure is large in size, it requires a large scale distributed FOS system to acquire a comprehensive monitoring of the structure; whereas if the structure is not large enough, effective monitoring can be achieved using quazi-distributed sensors[32]. There also exists another classification based on sensing capabilities such as intrinsic and extrinsic sensors[33] where these sensors are based on modulation or demodulation and these sensors could be physical or chemical or biomedical. These sensors can be organized into continuously distributed or quazi-distributed systems. Finally classification can also be based on the type of mountings (i.e., either surface bonding or embedded), where these sensors can be intrinsic or extrinsic. Thus, the classification is not universal but varies depending on type of sensing, mounting, distribution and type of application. Most of the current sensor designs are 'wavelength or frequency modulations' based such as fiber Bragg gratings (FBG) and 'phase modulations' based such as interferometric sensors[34,37]. Some sensor designs such as those based on intensity modulations have been developed and effectively employed in civil engineering applications[35]. The following sections will present some sensors typically useful for most of the engineering and medical applications.

\section{Intensity modulated or distributed fiber sensors}

Intensity modulated sensors are based on the modulation of light intensity (amplitude) in the fiber. This kind of sensor can measure any parameter that can cause intensity losses in the guided light beam. Sensors that vary the intensity of light are the simplest as only a source and detector are required. The advantages of these sensors are: 
simplicity of implementation, low cost, possibility of being multiplexed[38] and ability to perform as real distributed sensors. Fracture losses or local damages in a structure cause light intensity variations. Its main application is for fault finding and attenuation monitoring in optical networks. As these sensors can be distributed, they can be also suitable for the large structural applications, since all the segments of an optical fiber act as sensors, and therefore, the perturbations within various segments of the structure can be sensed.

There exists two major distributed sensor methodologies, the first one is the optical time domain reflectometry (OTDR) and the next one is Brillouin scattering. In the OTDR, Rayleigh and Fresnel scatterings are used for sensing structural perturbations. It is not necessary to study about the scattering phenomena in detail as it works on simple light scattering principle. For example the OTDR relies on the reflection of light that has been launched into a fiber from an amplitude-modulated and pulsed source. Using the OTDR technique, from the backscattering of the light, it is possible to obtain the value of the light intensity along the whole fiber, by measuring the time of flight of the returned pulses. In this way, it is possible to detect losses in the fiber and to locate these losses with quite good spatial resolution. Distributed sensors have a great potential in civil engineering due to their inherent distributive nature[32], but they also possess certain limitations such as insufficient resolution, weak detectable signal and cumbersome demodulation system. Moreover as only relative variations in the intensity of the light source are measured and these variations lead to false readings, unless a referencing system is used[39].

\section{Spectrometric or quasi-distributed fiber sensors}

The spectrometric sensors monitor changes in the wavelength of the light. These sensors are not as sensitive as the interferometric sensors (see later section) but their configuration, installation, and data processing are extremely easy. An advantage of these sensors is that the sensed information (shift in wavelength) is an absolute parameter, and thus obtained absolute measurements are more reliable as compared to relative ones. The wavelength-encoded nature of the output also permits ease in multiplexing. However, here intermittent segments of fiber act as sensors and such segmental formation is achieved by fiber gratings[22]. Intensive study on fiber gratings began after a controllable and effective method for their fabrication was devised by Meltz et al[40]. In recent years, there have been a number of research initiatives towards the development and deployment of FBG based sensors for sensing applications in civil and structural engineering[41-43,5].

A Bragg grating is a periodic structure, fabricated by exposing a photo-sensitised fiber to an ultraviolet light. Several gratings can be placed along a single optical fiber in an intermittent way to obtain a quasi-distributed sensing system. When light from a broad band source interacts with the grating, a single wavelength, known as the Bragg wavelength, is reflected back whereas the rest of the signal is transmitted. An external mechanical strain in the fiber (a stimulus) shifts the Bragg wavelength (response of the fiber) through expansion/contraction of the grating periodicity. Similarly, a temperature variation (stimulus) causes thermal expansion/contraction of the grating periodicity and also changes the refractive index (responses). These effects provide the means of employing the FBG written fibers as the sensor elements for measuring strains and temperatures (Fig. 1b; $[44,40,45]$. When light from a broad band source interacts with the grating, the Bragg wavelength is reflected back whereas the rest of the signal is transmitted. The Bragg wavelength $\lambda_{b} \quad\left(=2 \Lambda n_{e f f}\right)$ depends both on the physical characteristics of the fiber and on the geometrical characteristics of the grating. $n_{\text {eff }}$ is the effective refractive index of the mode propagating along the fiber and $\Lambda$ is the period of the FBG. Both the effective refractive index and the grating period vary with changes in strain $\Delta \varepsilon$, a temperature change $\Delta T$ and a pressure change $\Delta P$, imposed on the fiber. An applied strain and pressure will shift the Bragg wavelength through expansion or contraction of the grating periodicity and through the photo elastic effect.

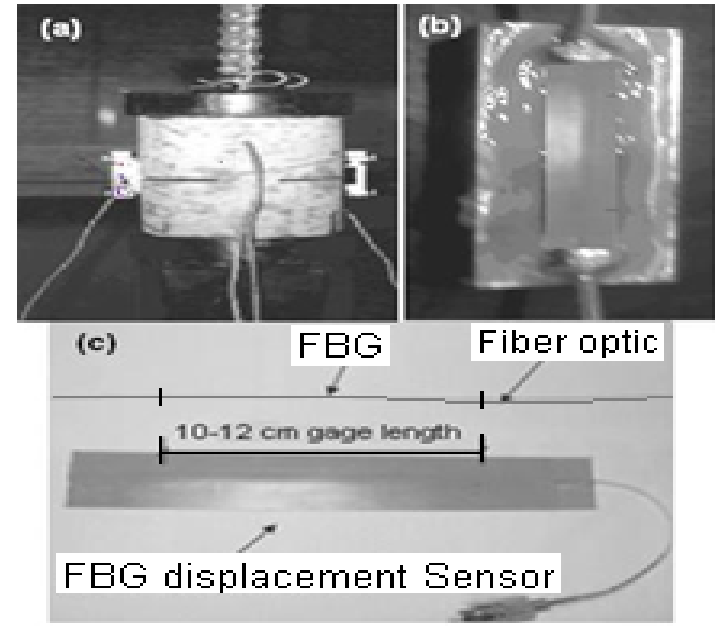

Figure 2. Embedded and surface bonded FBG sensors (a) Embedded FBG in concrete specimen; (b) Surface bonded FBG on beam; (c) FBG fiber with protecting casing strip

The strain of the FBG, which can be due to strain in the host material (in which it is embedded or surface bonded on) is detected as a shift in the wavelength of the reflected light[16]. These devices have a number of distinct advantages over other sensing schemes. One of the most important of which is that the sensed information is encoded directly into wavelength, which is an absolute parameter. Therefore the output does not depend directly on the total light levels; losses in the connecting fibers and couplers, or recalibration or re-initialization of the system. In addition, wavelength division multiplexing is readily achieved by fabricating each grating at a slightly different frequency within the broad-band source spectrum. FBG sensors (Fig. 2), which can be easily multiplexed at various 
locations to measure strains, are thus effective quasi-distributed sensors. For SHM where the potential damage or leakage locations are unknown, it is difficult to pre-determine the places to install FBG sensors but they are excellent in localizing the damage only when the specific area(s) of interest are known. They can be applicable in aerospace and civil industry including bridges, buildings, and dams[46-47] for measurement of dynamic strain, temperature, wind or water pressure, and damage location[48-49]. In fact in civil engineering Indus- try, FBG based strain sensors have been proven to be attractive alternatives to the conventional electrical strain gauges[50]. They were also used for hydrogen sensing in chemical industry[51].

\section{Intrinsic and extrinsic sensors}

In general, the FOSs uses optical fiber either as the sensing element ("intrinsic sensors") where fiber is used as the input/output medium, or as a means of relaying signals from a remote sensor to the electronics that process the signals ("extrinsic sensors"). In the intrinsic sensors, the measurand directly acts over the optical fiber and changes some physical properties of the fiber. These changes on the fiber produce a change on the properties of the light (intensity, phase, polarization, wavelength or transit time of light) traveling inside the fiber and the light never exits the fiber[39] to measure strain, temperature, pressure, electric voltage etc. Sensors which modulate light intensity use mainly multimode fibers, but for other parameters only single mode cables are used. For example a simple accelerometer (sensor) can be made by placing a mass subject to the acceleration on a multimode fiber. The force exerted by the mass on the fiber causes a change in the intensity of light transmitted. If required these intrinsic sensors can be used as distributed sensors up to 1 meter.

Applications of intrinsic sensors: All parameters like temperature, displacement, pressure, $\mathrm{pH}$ and smoke variations can be measured effectively by observing refractive index variation. Temperature can be measured by using a fiber that has a dominant loss mechanism known as evanescent loss[52] where the fiber will have two different core and cladding temperature coefficients. Electrical voltage can be sensed by nonlinear optical effects in specially-doped fiber, which alter the polarization of light as a function of voltage or electric field. Sensors based on the Sagnac effect are used for angle measurement[53]. FBGs are very effective intrinsic sensors for measurement of strain or temperature as presented previously. Recently these FBGs have also been developed to measure co-located temperature and strain simultaneously with very high accuracy[54-55]. Even the distributed sensors (like Brillouin scattering based) are also effective intrinsic sensors; they were used to detect strain and temperature over larger distances of about 20-30 kilometers in the past. Other intrinsic sensors were used as hydrophones (distributed sensors) for seismic, sonar applications[56] and as temperature and pressure sensors for oil industry[57]. Hydroph- ones are fiber sensors with more than one hundred sensors per fiber cable. Depending on the arrangement of sensors in different arrays, hydrophone systems may be named as bottom-mounted or towed streamer systems etc[58]. These systems can work at very high temperatures suitable for semiconductors[56]. Some other intrinsic sensors were used as interferometric such as fiber optic gyroscopes for aerospace[59], land transit for navigation purposes and hydrogen sensors. Some other miscellaneous intrinsic sensors in electrical engineering are used for detection of electromagnetic fields (mega hertz)[60], measurement of $\mathrm{AC} / \mathrm{DC}$ voltages, electrical power[61,62], used in electrical switchgear to transmit light from an electrical arc flash to a digital protective relay[63].

In the extrinsic sensors, mostly multimode optical fiber is used to transmit modulated light from either a non-fiber optical sensor, or an electronic sensor connected to an optical transmitter in which the optical fiber is used purely as the input/output path. The fiber only carries the light from the source to the sensing part, and from the sensing part to the demodulation system. Extrinsic sensors are used to measure vibration, rotation, displacement, velocity, acceleration, torque, and twisting[39].

Applications of extrinsic sensors: A major benefit of extrinsic sensors is their ability to reach places which are otherwise inaccessible with excellent protection of measurement signals against any noise[64]. There are many applications such as the measurement of temperature inside aircraft jet engines by using a fiber to transmit radiation into a radiation pyrometer located outside the engine. Extrinsic sensors can also be used in the same way to measure the internal temperature of electrical transformers, where the extreme electromagnetic fields present make other measurement techniques impossible.

\section{Micro bend Sensors}

An intensity modulation multimode sensor, where the modulation caused during measurement of any property like pressure can be reflected in the form of a micro bend loss modulation, moving fiber modulation or an absorbing layer modulation. The sensor comprises of a certain length of fiber which is placed between two rigid plates having an optimum corrugation profile so that the fiber experiences multiple bends. Due to the micro bending induced losses, the lower order guided modes are converted to higher order modes and are eventually lost by radiation into the outer layers resulting in a reduction of the optical intensity coming out of the fiber. A displacement of the plates due to measurable property causes a change in the amplitude of the bends and consequently an intensity modulated light emerges from the fiber core. Such sensors are apt for civil engineering structures especially in embeddable composites like reinforced concrete material where the reinforcing fibers in the composite structure act as natural bend loss sites for the optical fiber. In addition, the micro bend sensitive optical fiber can be jacketed in such a way that periodic micro bending is induced. When the jacketed fiber 
is loaded, the overall effect leads to an increase in loss. They were also used effectively in situ device for salinity measurements (chloride detection) of ocean surface[65]. The combination of multiple micro bend sensors can form a sensor array for the quasi-distributed sensing application in the monitoring of local strain or deformation along structures, and the OTDR can be conveniently used for interrogation of each sensor unit. The sensor sensitivity can be set at a specific value according to the requirements of the measurement condition. Connected with multiplexed sensing processing schemes, the sensor array may find an application in the real-time monitoring and damage detection of large and critical engineering structures[66].

\section{Inferometric fiber sensor}

Most of the components of these sensors use either all-fiber or integrated optic material to provide better stability and compactness. An interferometric sensor works on the modulation in the phase of light emerging from a single mode fiber. The variation in phase is converted into an intensity shift using interferometric schemes (Sagnac forms, ring resonators, Mach-Zehnder, Michelson, FabryPerot or dual mode, polarimetric, grating and Etalon based interferometers.). These sensors can be used as intrinsic or extrinsic sensors and distributed or quazi-distributed sensors depending on the type of applications. The applications are in acoustic (e.g. hydrophones), rotation (eg., gyroscope), chemical, biological etc[67-69] to measure strain, temperature etc. All these schemes are used to split the light wave and then recombine at a photo-detector[70]. There are many applications of in several sensing systems[71-74] such as sensing vibration signals[75]. Recently a long FBG based Michelson modal interferometer is studied as a sensing structure for measuring environmental refractive index, temperature, and liquid level by Caldas et al[76].

\section{Some Selected Applications of Fiber Sensors}

The fiber sensors are used in past few decades and can be used in future in many fields, and their applications range from space to undersea. i.e every where were the light/data communication is feasible.

1) Transmission: In the chronological order, it is one of the first areas where optical fibers were used. The first theoretical prediction of practical applicability of optical fibers occurred in 1966, system experiments in 1976, and economically practical system deployments in 1980[77] . The "optical fiber or light wave communication" have the advantages of being accurate, descriptive and more readily understandable. In telecommunications, glass fibers that carried light wave communications were called "light guides". In recent years it has become apparent that these light wave systems are steadily replacing copper wire as an appropriate means of communication signal transmission.
The only basic difference is that the fiber-optics use light pulses to transmit information down fiber lines instead of using electronic pulses to transmit information down the copper lines. They span the long distances between local phone systems as well as providing the backbone for many network systems. These systems are also useful in cable television services, university campuses, office buildings, industrial plants, and electric utility companies.

2) Power Transformer hot-spot temperature measuremen $-t$ : Fiber-optic techniques were first used to measure winding temperatures in power transformers more than 30 years ago. Knowledge of the hot-spot temperature in a power transformer is a key element for its design and utilization. It enables manufacturers to refine their design, improve the quality and competitiveness, and users to utilize the full overload capacities of transformers and hence meet requirements of their customers without excessively reducing life expectancy of equipment[78-80]. The measurement of hot-spot to allow predictive loading and enhance capacity utilization has increased giving rise to more and more cost- effective fiber-optic temperature measurement systems such as the fluorescent decay time technique has been developed. These technologies offer an opportunity for direct hot-spot measurement without any detrimental effects to the dielectric integrity of the transformers. Additionally, the main source of power to industries or households or trains have to be monitored on real time basis, there safety especially during times of cyclone or earthquakes or tornadoes is crucial. Any physical problems in the transformers like dis-integraty, cracks in long range power transmission cables. Fractures in joints including direct buried cable, damages in cable tunnels, cracks in cable ducts or ancillary switchgear and other ancillary equipments can cause severe disruption (failure in power transmission network) to supply as well as reduce equipment lifetime. Additionally, if there is over head network (power cables), can result in sag or sudden short-circuiting. Thus, the fiber sensors for example like Sensornet's Digital 'Thermal Profiling', 'Condition Monitoring', 'Strain Profiling' and Dynamic Cable Rating technology[81] offer a continuous snapshot of all these equipments for maximum efficiency and structural integrity. Moreover Sensornet provided a Sentinel DTS-XR Distributed Temperature Sensing system for monitoring the transmission network. The Sentinel DTS-XR provides world beating performance and can measure with high resolution at distances of $30 \mathrm{~km}$.

3) Real time based complete monitoring of Aeroplanes/ spacecrafts: The term 'complete' refers to an integrated network of sensors, which could monitor not only structural elements, but also the health of electronics, hydraulics, avionics, and other systems in aeroplanes. All types of sensors like extrinsic, intrinsic distributed and quazi distributed sensors are adoptable for aerospace structures depending on need, parts of craft and type of monitoring. For example, Lee et al[82] demonstrated the applicability of 
FBG sensor system for wings quite effectively. Networks of sensors mounted on commercial aircraft might one day check continuously for the formation of structural defects, possibly reducing or eliminating scheduled aircraft inspections[83] like nerve endings in a human body, in-situ sensors offer levels of vigilance and sensitivity to problems that periodic checkups cannot[19,84]. Adoption of effective sensing system can greatly reduce maintenance expenses for commercial aircraft. Those costs are rising as the aircraft age, many well beyond their design lifetimes. Ground crew technicians might plug a laptop or diagnostic station into a central port on the aircraft to download structural health data. Eventually "these sensing systems" fitted with many sensors could self-diagnose and signal an operator when repairs are needed. The potential locations on aircraft where the fiber sensors can be placed and diversified monitoring parameters can make aerospace a very challenging field for adaptation of fiber sensors. For example the harsh environments in which these aerospace structures have to work are the major limit for the employment of standard fiber optic sensors for the 'thermo-mechanical' monitoring processes. Thermal loads which act on these structures do not allow the use of standard fiber optic sensors used for classic avionics application. In fact, many aerospace structures can be exposed to temperatures up to $1000^{\circ} \mathrm{C}$, higher than the operation temperature of the standard fiber optic sensors. Latini et al[85] used a tunable laser source, to easily measure the spectral response of different fiber sensors intended to monitor thermal process. This allows performing a multi-sensor interrogation and analyzing many physical parameters, such as: temperature, strain, pressure, etc[20]. In particular they used temperature resistant Fiber Bragg Grating sensors; they were proved to be successful even up to $600^{\circ} \mathrm{C}$. They have resulted in a good response in terms of: sensitivity, resolution, repeatability and dynamic range of the measurement were obtained. The NASA contractors and research collaborators in United States are aggressively working to develop newer sensors, and it was reported that the latest fiber structure for use in NASA is of a size of human hair.

4) Real time monitoring of ships: There are two factors here, the first one is the monitoring of ship movement in water/sea and the second one is the monitoring the ship itself for defects. In developed countries like United States, the authorized security (navy) uses fiber optics to secure ships in water. This includes logistical trail stretches back from the battle or disaster to supply bases in the U.S. Most of those supplies travel by sea on ships under the Military Sealift Command. During a war, more than 95 percent of all equipment and supplies is carried by sea going vessels and thus better access control and perimeter security needs to be provided. The technology includes fiber optic modules from Weed Instrument Co[86]. Even in other countries, the fiber sensors for ship monitoring are increasing, as US type models are adopted in several countries.

For the second factor, i.e for monitoring defects in the ship, fiber sensor especially FBGs[87] are as apt as they are for any other engineering structures. Recently in 2001, embedded FBG technology was adopted for a Norwegian naval vessel by Wang et al for composite ship hull monitoring. Signal processing is key for real-time structure monitoring, and they have presented the modular signal processing system effectively. The other sensors which can be used are BOTDR strain sensors, as they have better efficiency due to good resolutions in signals[88]. They can be used for defect identification by measuring strains and also for detecting changes in water levels outside the ship. The future of FBG or BOTDR sensors are very promising for ships/vessels as it can help in prognosis of remaining life and save huge money for governments.

5) Real time monitoring of submarines and deep sea floor: The issues and solutions adopted for monitoring of ships can be used in as is conditions [88-89] even for monitoring submarines except that the FOS systems should have thorough water protection schemes.

However, inorder to monitor deep sea floor[90], two factors, i.e, telemetry method and power supplying system, are important. There are two major technologies to understand real-time geophysical monitoring on a deep-sea floor. The tethered buoy-satellite system is one such technology. Even if a giant buoy system with strong moorings is used to maintain its reliability during extremely rough weather, it may be not strong enough. Other factors such as antenna direction heading to a stationary satellite, communication method between ocean floor and a surface buoy, and power sources for bottom equipment, are also difficult tasks to be overcome. During few decades, only a weather buoy, which took measurements at only the ocean surface, has been successful[91-92]. Another approach is to use submarine cable systems. The submarine cable system has long technological history and has been proven in the field[93]. Although optical fiber submarine cables are one of the most advanced and reliable technologies, the use of such submarine cables is extremely costly compared to free-fall-pop-up systems. Another kind of submarine cable is an analog coaxial cable, which still provides electrical power and real-time data-telemetry similar to optical fiber systems. If real-time measurement is mandatory for a particular observation such as likely for earthquake and tsunami monitoring, submarine cables would be the best choice. However, the fast growth in the field of fiber sensor will surely make them adoptable as the cost of these fibers is likely to reduce as advent of mass production. Someday, real-time geophysical observatories on a deep-sea floor could be realized with high reliability and at a reasonable cost.

6) Real time monitoring of refinery process: Crude unit overhead line corrosion is one of the most serious problems in oil refineries[94]. Changes in process, environmental conditions, or feedstock that lead to $\mathrm{HCl}$ condensation are the main causes of overhead line corrosion. Corrosion is one of the major problems that affect the integrity of pipelines in refineries. All pipelines that transport petroleum products experience wall thinning and internal corrosion 
that are associated with the chlorides and sulfides in the products they carry. Buried pipes are often affected by external stress corrosion cracking due to the combined effects of the surrounding soil environment and pressure fluctuations. The procedure used to refine oil products requires careful attention to the temperature and pressure as well. In addition, by their very nature, refineries make use of inflammable materials that could be exposed to direct sunlight during the daytime. In order to ensure the safety of the plant, systems must be well-designed so that sparks cannot reach inflammables, and it is necessary to use rugged devices for the monitoring and management system. The control and monitoring system can comprise of effective fiber optic network as the communication network between key devices and the control center to also support voice, video monitoring, and security infrastructure[95].

7) Real time monitoring of subsea, oil and gas industries: more or less the same factors discussed in monitoring refineries like corrosion, pressure and temperature[94-96] are vital, and the fibers like FBG[97] or BOTDR are apt even for monitoring of subsea. However, there are many more vital factors [96-98] like (a) Process conditions of oil and gas industries: Temperature, pressure, flow and composition. (b) Environmental conditions: Sea water temperature, pipeline spans, stress / strain at spans and landslide areas. (c) Required type of sensors: Pressure, temperature, strain gauges. (d) Sea water: composition / flow. (e) Location of sensors: Low spots, along pipeline at "selected" locations. (f) Dynamic simulation model of: Wells, subsea equipment and flow lines, multiphase pipelines, parts of the shore processing plant, real time model interfaced to vendor control system for online monitoring of slugs, tracking, offline planning / training simulator, etc.

Once these factors are understood, then the aligning of fiber senors can be achieved for monitoring subsea, oil and gas industries. Additionally as presented by Brower and Prescott[98], a smart pipeline technology can be introduced for real-time monitoring. The smart pipeline technology allows for auto-adaptive measures to ensure trouble free operation of the entire pipeline system. Real-time monitoring and flow control issues drive the development of smart pipelines in oil \& gas reservoirs for both onshore and offshore deepwater environments. A key feature of this technology is to develop full knowledge of flow assurance parameters in pipelines etc. A methodology to offer smart pipeline technology, including real time, on-line monitoring and control system for subsea production and pipelines was presented by Brower and Prescott[98]. The instrumentation was based on fiber optic technology. The system included problem prevention or mitigation with early detection and proactive intervention by applying new developments in the field of optic fiber monitoring. Such a system was deployed in deep water projects in the Gulf of Mexico. This had resulted in the development of a smart field control system, whose flow chart is as shown in Fig. 3 with automated data analysis and response. There are a number of operation and mechanical parameters which may be monitored or derived using this data system. The data acquired may be used to predict the onset of problems hence allowing timely corrective action. The measurement features of the new smart pipeline method included, real time data collection and modeling, dynamic and static strain measurement, vortex induced vibration, touchdown zone monitoring, stress concentrations, continuous or discrete temperature and pressure measurement along the entire system. Additionally, cryogenic temperature and structural monitoring in liquid natural gas (LNG), slug and anomalous flow, integration with smart well systems, integration with subsea processing, ultrasonic discrete measurement.

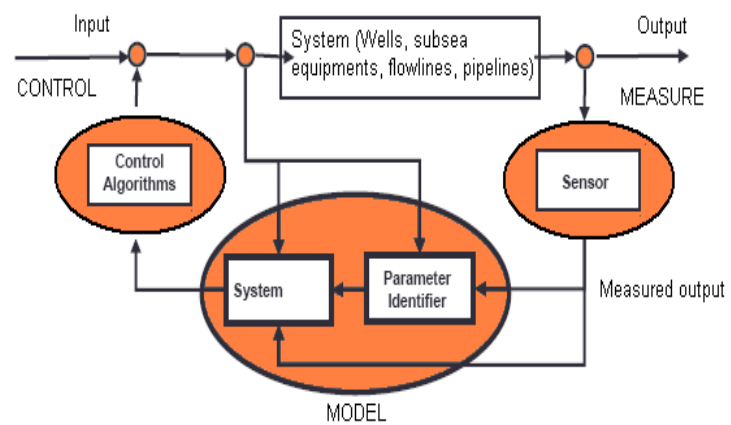

Figure 3. flow chart of Fiber sensor application
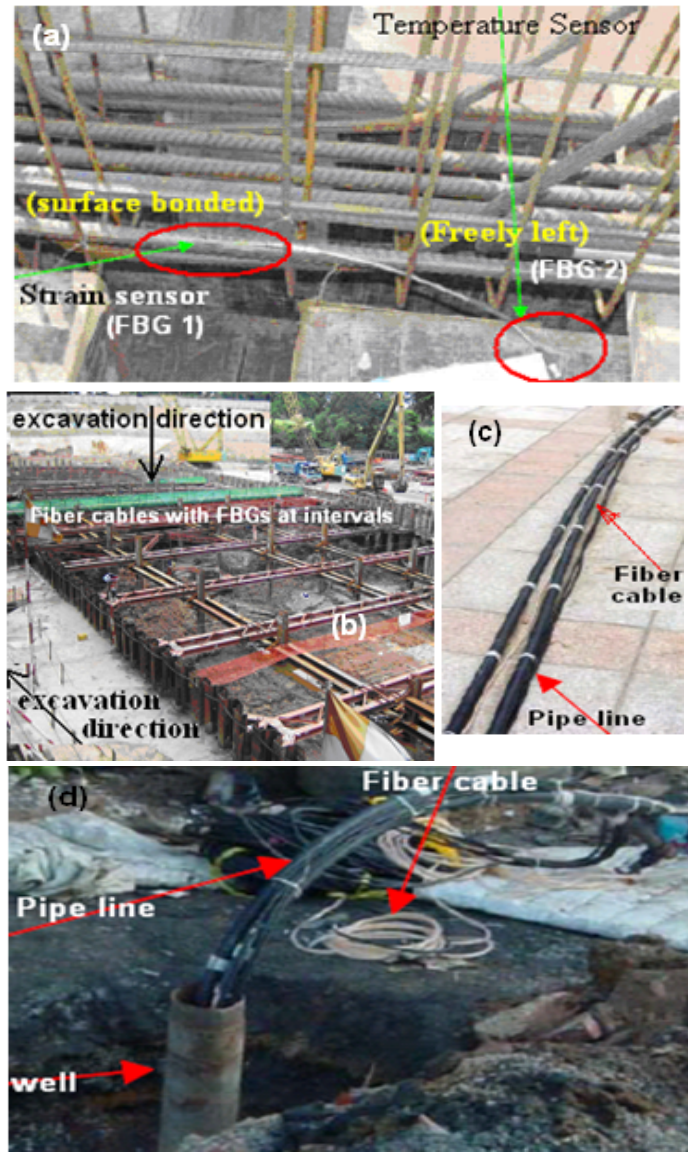

Figure 4. Practical applications of FBG sensors (a) For bar dressing; (b) Monitoring temporary excavation support structures; (c) Pipe line fitted with fiber; (d) Monitoring pipe lines 
(8) Real time monitoring of civil engineering structures: After almost two decades of development, SHM has currently become the highlight of researches and applications in vast civil infrastructures (Fig. 4) all over the world[99]. And its core is damage detection and identifycation. As it is a big challenge to perform accurate damage analysis, especially the damage location, via global information. Local damage monitoring seems paramount as local damage can be anything which disturbs the integrity like a crack, fatigue, slip, debonding, effective forceresistance area loss, and so on. Strain is an alternative parameter which can be used to describe deformation, study the crack opening and even detect the slip and bonding, so high-quality strain sensor has always been pursued by the structural researchers[100]. Several types of fiber optic strain sensor have been developed, including those based on intensity, polarization, interferometry especially FabryPerot interferometric, and FBGs[101-102].

The infrastructures are generally large, long span and serve for a very long time, so the local durable and reliable sensors are the foundation of successful health monitoring systems. FOS system is used and will be used in various key civil structures, including buildings, piles, bridges, pipelines, tunnels, dams, soil excavation etc[103-104,6]. Additionally, they can be used for various materials like concrete, steel or composites used in civil engineering.

(9) Monitoring of chemicals, heritage culture, food and health care. The fiber sensors are not just adoptable to solid structures and gases but were also used for chemicals, polymers and human body.

(i) DNA sensor: The first integrated fiber optic DNA sensor array capable of simultaneously monitoring multiple hybridization events has been prepared by Healey et al[105]. In this study, DNA probes were covalently immobilized by reaction with succinimidyl ester residues in acrylamide-based polymer matrices. DNA sensor arrays of three different oligonucleotide probes, $\mathrm{p}(\mathrm{dA})(18-\mathrm{mer})$, H-ras wild-type (10-mer), and H-ras mutant (11-mer) were fabricated, and real-time hybridization of fluorescein isothiocyanate (FITC)-labeled target oligonucleotides to the array was monitored. H-ras wild-type and H-ras mutant differ by a one-base substitution (point mutation) and were used to demonstrate the sensor's ability to distinguish point mutations. Thermal studies indicated a $13^{\circ} \mathrm{C}$ decrease in the $\mathrm{Tm}$ of a duplex with a single-base mismatch. The fiber optic DNA sensor array was used to discriminate a point mutation by monitoring the real-time hybridization of FITC-labeled target oligonucleotides at $54{ }^{\circ} \mathrm{C}$ and detected labeled-target oligonucleotides in the range $0.2-196 \mathrm{nM}$. The lower detection limit is approximately an order of magnitude lower than previously reported DNA biosensors. The DNA sensor array was used to positively identify a point mutation of a biotin-primer-labeled (109 bp) PCR product of the H-ras oncogene. The unitary fiber optic sensor array is highly sensitive, has the ability to determine point mutations, and has the potential to sample submicroliter volumes due to the small volumes of the individual array elements $(20 \mathrm{pL})$.

(ii) Hydrogen sensor: A decade ago, Sutapun et al[106] developed a new type of optical hydrogen sensor with a FBG coated with palladium thin film. The sensing mechanism in this device is based on mechanical stress that is induced in the palladium coating when it absorbs hydrogen. The stress in the palladium coating stretches and shifts the Bragg wavelength of the FBG. Using FBGs with different wavelengths many such hydrogen sensors can be multiplexed on a single optical fiber. Moreover, hydrogen and thermal sensitivities of the sensors were calculated using a simple elastic model. They have demonstrated multiplexing of two sensors successfully. Additionally, to quantify the amount of stress in the palladium film as a function of hydrogen concentration, a novel and very sensitive method was devised and used to detect deflections in a Pd-coated cantilever using an evanescent microwave probe.

(iii) Heritage culture and food sensors: The study of sensors for food and culture was extensively studied by Mignani's group[107,3]. A demand for fresher, better-tasting, safer, healthier and higher quality food is escalating in every country. We live in a global marketplace, and we eat a great variety of foods from many different countries. These are grown using a variety of production practices, from small organic operations to large-scale mechanized farms. On the one hand, product buyers want to be reassured that farmers are taking reasonable steps to ensure that the produce delivered is safe and free from human pathogenic bacteria and mycotoxins. On the other hand, consumers and food handlers (restaurateurs, retailers, etc.) need to know that the food which they eat and serve is safe and healthy. Sensors based on optic and micro-optic techniques are found to offer effective and low-cost solutions for many industrial and process control applications. Especially in the case of spectroscopy-based devices, the intrinsic optical and mechanical characteristics of optical fibers, together with the wide availability of bright LEDs and portable spectrometers, have made it possible to implement compact instrumentation with a high potential for many applications in the food sector. Various spectroscopy-based fiber-optic and micro-optic devices have been designed and tested for monitoring the quality and safety of typical foods, such as extra virgin olive oil, wine, whisky, beer, tequila, honey, milk, and many others. These sensors belong to two different types: those performing direct spectroscopy on the food sample, and those making use of an optical transducer which changes color or fluorescence according to the sample under test[3].

(iv) Health care sensors for human body: There are various types of fiber chemical sensors, fluorescent probes and bioassays and many biosensors[108] for use in healthcare and/or in chemical or drug process for medical practices. Mignani and Baldini[109] presented an over view of FOSs for vivo biomedical monitoring and a comparison to the performance of traditional devices were performed. Biomedical FOSs are attractive for the measurement of 
physical, chemical and biochemical parameters and for spectral measurements directly performed on the patient. Later Mignani and Baldini[1] have presented a study with particular attention paid to the advantages that these sensors are able to offer in different application fields such as cardiovascular and intensive care, angelology, gastroenterology, ophthalmology, oncology, neurology, dermatology and dentistry. Spillman et al[110] proposed a 'smart' bed to non-intrusively monitor patient respiration, heart rate and movement using spatially distributed integrating multimode FOSs, which can be very useful for elderly population. The sensing fiber was integrated into a bed and test subjects were monitored in different positions. The sensor outputs were then correlated with subject movement, respiration rate and heart rate. The results indicated that the inter-modal sensor could detect patient movement and respiration rate while the mode conversion sensor could detect patient movement, respiration rate and heart rate. The other sensors[111-114] are 1) immunodiagnostic sensors 2) $\mathrm{pH}$ sensors 3) biomedical sensors. All these sensors are completely passive they pose no electrical shock threat to the patient. The future of these sensors is promising in different application fields such as cardiovascular and intensive care, angiology, gastroenterology, ophthalmology, oncology, neurology, dermatology and dentistry.

\section{Protection of Fiber Sensors}

FOSs have to be surface bonded on or embedded in different host materials subjected to various conditions. Therefore, sensor interaction with host structure is of great importance especially if monitoring engineering structures or sub sea or construction. Effective sensing demands a compliant mechanical contact between the FOS and the host structure to ensure an appropriate transfer measurands of interest into light signals. However, an additional protection has to be used to isolate the delicate bare fiber from water and alkaline and from damages caused by the host structure. The protection scheme of the fibre in the sensing region differs with different host materials and type of bonding (i.e embedding or surface bonding). Experiences from recent applications showed that polymide coated fibers and epoxy were possible to secure an excellent mechanical coupling between the fibres and the anchorages in concrete structures[115]. In addition, the thermal expansion coefficient of the sensor packaging should be approximately equal to that of the host structure to avoid possible slippage between the interfaces. Moreover, the installation of FOS especially Bragg Gratings on base structures requires longitudinal uniform bonding. Otherwise, the non-uniform bonding of the gratings will cause several reflected Bragg wavelength peaks when the gratings are strained. In such a situation, accurate strain measurement will be impossible. It was observed that the survival rate of FOSs during installation was about $75-90 \%$. Therefore redundant sensors should be used for critical measurements and careful planning is required. Especially, during installation, all-fibre compon- ents and connections should be delicately handled. Mechanical and thermal fatigue as well as chemical aggression will also decrease the life of FOSs. Special attention and various measures have to be taken to relieve these adverse effects on optical fiber health monitoring. Additionally for surface bonding FBGs, care has to be taken that the bonding layer employed between the FBGs and the host structure should be highly durable and does not peel off during the monitoring period.

\section{Present and Future Generation Sensors}

Infrastructures, structures carrying liquids or gases, communicational systems, telephone lines, tornadoes or tsunami or cyclone warning systems etc are important facilities of modern day. Some of the natural calamities like Tsunami in South East Asia (2004) have raised the question of safety of 'under sea or over sea communication lines' or 'oil industries' or 'marine life'. Some of the failures like space shuttle Columbia's explosion on Feb 1, 2003 have raised questions of safety in aerospace/ space craft industry. Some of the civil engineering structures failures like Mississippi river bridge collapse on August 1, 2007 have raised concern about safety of civil structures. Thus health monitoring has attracted the effort of many engineers throughout the world and is fast emerging as a pioneering field.

In the last couple of decades, abundant 'types of fiber sensors' are manufactured, re-manufactured with improved quality (year after year) and used for various fields $[107,116$, Figure 5]. Thus the future of these fiber sensors is very promising, for example newer Brillouin FOSs have found its applications in many niche engineering and biomedical fields with better performance than fiber sensors of older genre sensors. These Brillouin sensors are effective even for long ranges ( $>10$ meters) and large areas to measure various parameters like temperature and strain along a single mode fiber[117] with high resolution quality either separately or simultaneously. The distributed Brillouin sensors can be used for much broader coverage (than FBGs) and can locate fault points not known prior to sensor installation. Unlike competing sensor technologies, Brillouin systems directly leverage the economies of scale from the millions of kilometers of fiber optic telecommunications fiber installed worldwide. There are two types of Brillouin sensors, first one is Brillouin Optical Time Domain Reflectometers (BOTDR) based Brillouin scattering of a single pulse. Second one is Brillouin Optical Time Domain Analysis (BOTDA), which uses a more complicated phenomenon known as Stimulated Brillouin Scatter (SBS). The BOTDA technique is significantly more powerful as it uses enhanced Brillouin scattering through two counter-propagating beams. The primary advantage of BOTDR technology is that only one end of the fiber needs 
to be accessible. Both these types of Brillouin fiber sensors were employed successfully due to their accuracy in measurement of various measurands like strain and temperature[118], for monitoring of various structures, components and parameters like pipeline buckling[119], pipeline corrosion[120], power transmission lines[121], crack detection by separate measurement of strain and temperature[122] and their simultaneous measurements[123].

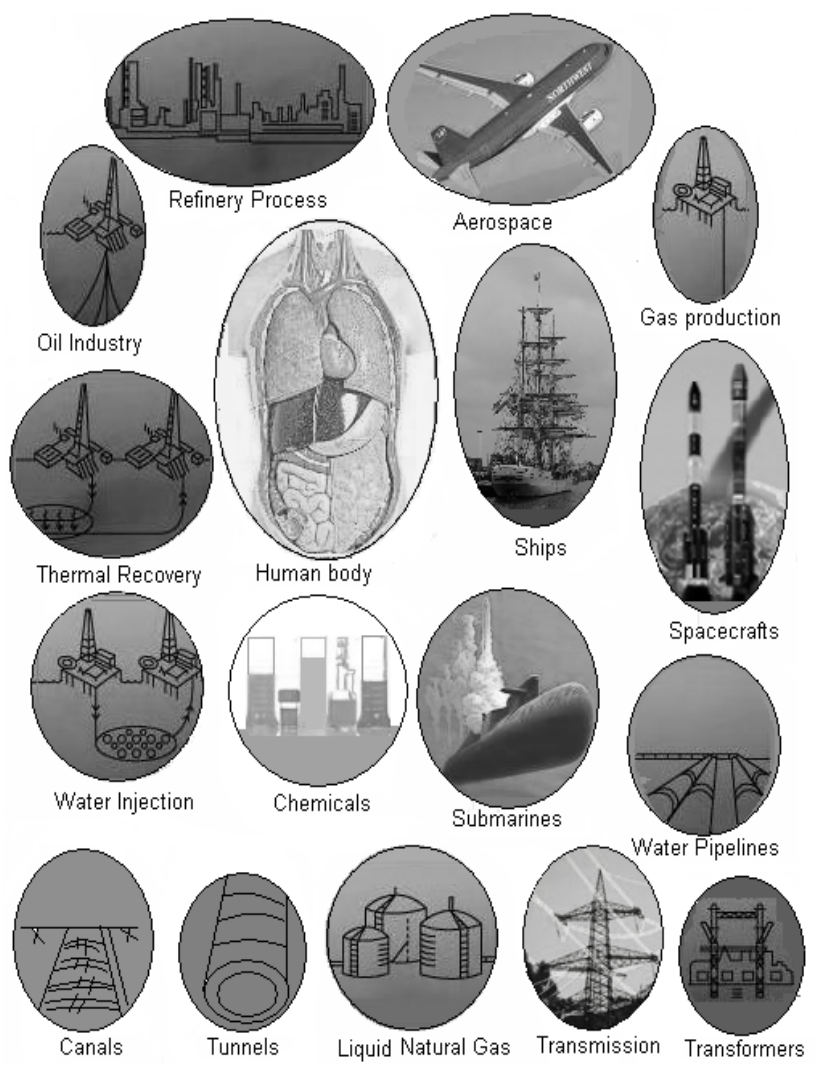

Figure 5. Fiber sensor applications in various engineering fields

In general the classifications and types of fiber optic sensors are purely authors and manufacturer specific but for end users they are sensors which probably act as control system i.e FOS system should sense and activate/trigger warning. An ideal example might be a highway (equipped with FOS system) that senses an accident and activates warning signs as a result. The FOSs are developed and used on basis of dual criteria, the first criterion is as a plausible replacement for conventional sensors to offer considerably improved reliability, performance, safety and optimized cost to the[124-125]. The second is the development and deployment of FOSs in new market areas.

For the first criterion, the inherent value of the FOSs system has to be sufficiently high in order to think for plausible replacement of existing sensor technology else there is a risk of possible continuation of existing technology by the end user. The following examples highlight the current evolution strategies in the filed of FOS for replacement of conventional sensors.

The fiber optic gyro, which is displacing both mechanical and ring laser gyros for few devices[12]. As this technology matures in the future it can be expected that the fiber gyro will dominate large segments of this market[126].

The electrical strain gauge that is used widely by structural engineers can be replaced by FBG sensors. These sensors[127-128] can be configured to have gauge lengths from $1 \mathrm{~mm}$ to approximately $1 \mathrm{~cm}$, with sensitivity comparable to conventional strain gauges.

The recent advances to reduce the weight of conventional sensors on flights, have given rise to human hair like sensors[81]. The Ikhana (NASA space program) uninhabited aircraft system is flying research missions with an advanced sensing technology installed on its wings that measures and displays the shape of the aircraft's wings in flight. In this mission, the new FOSs are located side by side with conventional sensors. However, the potential for weight reduction is one small part of the comparison which can increase new opportunities and applications that would not be possible with conventional technology. For example, the new sensors could enable adaptive wing-shape control the concept of changing a wing's shape in flight to take advantage of aerodynamics and make the aircraft more efficient. The NASA mentioned that six hair-like fibers were located on the top surface of the Ikhana's wings, which provided about 2,000 strain measurements in real time. The fibers are so small that they have no significant effects on aerodynamic lift and weigh less than two pounds. The FOSs are small that they could be embedded within composite wings in future aircraft. These sensors can be installed along periphery of the wings in future manned or unmanned flights.

Fiber sensors are being mass-produced is the field of medicine[1,111-114,129], as 1) immunodiagnostic sensors for measuring a wide variety of both high and low molecular mass analytes 2) $\mathrm{pH}$ sensors to measure blood gas parameters and dosage levels 3) biomedical sensors for the measurement of physical, chemical and biochemical parameters and for spectral measurements directly performed on the patient.

The second criterion is very challenging as it requires introduction of fiber sensors into areas where there are 1) no sensors in use (if any, they may be cheap sensors) or 2) ordinary hand usable instruments, which does not have capability for centralized sensing facilities or 3) no assessment to monitor the condition of the structure at a fast pace. The researchers around the world require constant inspiration to think about or identifying the areas (marketable) to introduce these FOS sensors for betterment of modern society and for our own safety. For example, the automotive industry, construction industry (especially to monitor excavation process) and other traditional users of sensors (especially low cost) remain relatively untouched by fiber sensors, mainly because of cost considerations. This can be expected to change as the improvements in optoelectronics and fiber optic communications continue to expand along with the continuing emergence of new FOSs. Recently in Singapore, for construction of mass rapid transit (MRT), FBGs were used to monitor excavation[55] and the 
purpose of using such sensors was explained to the Singapore government.

\section{Conclusions}

There are two important issues involved in health monitoring of any engineering structures or heritage culture or food or medicine. The first criterion is as a plausible replacement for conventional sensors to offer considerably improved reliability, performance, safety and optimized cost to the end user. The second is the development and deployment of FOSs in new market areas. The last two decades had resulted in optimization of components and prices with development of high quality sensors to replace traditional sensors for rotation, acceleration, electric and magnetic field measurement, temperature, pressure, corrosion, crack formation, acoustics, vibration, linear and angular position, strain, humidity, viscosity, chemical measurements and a host of other sensor applications. In summary, FOSs have been successfully applied to many fields such as gas and oil industry, and civil engineering structures, to monitor relative displacements, cracks, strain and temperature. Most of these applications as presented in this chapter utilize one or several of the significant advantages of Interferometer/ distributed/ quazi-distributed senors, for instance, their embeddability, ease of surface bonding, EMI immunity, and multiplexing ability, immunity to source intensity fluctuations (especially FBGs). These optical fibers are fragile and may be subjected to breakage during packaging and transportation, especially during installation under sea due to harsh water conditions. Hence depending on the nature of application, protection measures need to be taken. Wide applications of these sensors can be foreseen, not only in laboratory for proof of concept applications, but also in many practical situations as presented in this chapter like monitoring of bridges, pipelines, piles, traffic and excavation support structures.

\section{ACKNOWLEDGEMENTS}

Author likes to thank Mrs Radhika Madhav Annamdas for her assistance in drafting. The author would like to thank members of Laboratory of Monitoring Science, which is a global research platform for smart material applications. The author likes to acknowledge all researchers whose work is cited in this paper. Further information of the work and its accuracy can be obtained from respective researchers/ authors of the cited articles.

\section{REFERENCES}

[1] Mignani AG and Baldini F (1997). " Fibre-optic sensors in health care ",Physics in medicine and biology. 42(5):967-979

[2] Lamela H , Gallego D, Gutierrez Rand Oraevsky A (2011)
"Interferometric fiber optic sensors for biomedical applications of optoacoustic imaging, Special Issue: Laser Applications in Life Sciences, Journal of Biophotonics 4(3), 184-192

[3] Mignani AG, Ciaccheri L, Cucci C, Mencaglia AA, Cimato A, Attilio C, Ottevaere H, Thienpont H, Paolesse R, Mastroianni M, Monti D, Gerevini M, Buonocore G, Del Nobile MA, Mentana A, Grimaldi MF, Dall'Asta C, Faccini A, Galaverna Gand Dossena A. (2008). "EAT-by-LIGHT: Fiber-Optic and Micro-Optic Devices for Food Quality and Safety Assessment", IEEE Sensors Journal, 8 (7), 1342-1354

[4] Yang YW, Annamdas VGM, Wang C and Zhou Y (2008). "Application of Multiplexed FBG and PZT Impedance Sensors for Health Monitoring of Rocks" Sensors, 8, 271-289

[5] Kawabata Y, Kamichika T, Imasaka T, Nobuhiko Ishibashi (1989) "Fiber-optic sensor for carbon dioxide with a ph indicator dispersed in a poly(ethylene glycol) membrane", Analytica Chimica Acta, 219, 223-229

[6] Annamdas VGM, Yang Y and Liu H (2008). "Current development in fiber Bragg grating sensors and their Applications." Proceedings of SPIE, San Diego California, USA, 6932, 69320D (paper no: 6932-15)

[7] Fu H. Y.,Tam H. Y.,Shao L.Y,Dong X,Wai P. K. A.,Lu C., andKhijwania S. K. (2008) "“'Pressure sensor realized with polarization-maintaining photonic crystal fiber-based Sagnac interferometer", Applied Optics, 47(15), 2835-2839

[8] Francis TSY, Shizhuo Y, Paul BR (2008). "Fiber Optics sensors" CRC Press, Optical science and engineering, 132

[9] $\mathrm{Gu}$ B, Yin M J , Zhang A P, Qian J W, and He S (2009)"Low-cost high-performance fiber-optic $\mathrm{pH}$ sensor based on thin-core fiber modal interferometer", Optics Express, 17(25), 22296-22302

[10] Sumitro S, Tominaga M and Kato Y (2002). "Monitoring Based Maintenance for Long Span Bridges," First International Conference on Bridge Maintenance, Safety and Management, 14-17 July, Barcelona, Spain

[11] Weisenbach L(2008) "A report on fiber optic sensors", Report Code: IAS002D, BBC Research

[12] Udd E, Corones J and Laylor H M (1998) "Fiber optic sensors for infrastructure applications", Final Report, SPR 374, February 98, Oregon Department of Transportation, and Federal Highway Administration.

[13] Udd, E (1991). Editor, "Fiber Optic Sensors", An Introduction for Engineers and Scientists. John Wiley and Sons, New York, USA

[14] Kersey, A. D., Author, E. Udd, Editor. (1991). "Distributed and Multiplexed Fiber Optic Sensors", Fiber Optic Sensors: An Introduction for Engineers and Scientists. Wiley. New York

[15] Bartko H, Goebel F, Mirzoyan R, Pimpl W and Teshima M. (2005) "Tests of a Prototype Multiplexed Fiber-Optic Ultra-fast FADC Data Acquisition System for the MAGIC Telescope", 29th International Cosmic Ray Conference, Pune, India, 101-106

[16] Mrad N and Xiao G Z (2005). "Multiplexed Fiber Bragg Gratings For Potential Aerospace Applications," International Conference on MEMS,NANO and Smart Systems, Banff, 
Alberta, Canada July 24- 27, pp.359-363

[17] Ansari F (2007), "Practical Implementation of Optical Fiber Sensors in Civil Structural Health Monitoring," Journal of Intelligent Materials Systems and structures, 18(8): 879-889

[18] Yuan L and Dong Y (2008). "Multiplexed Fiber Optic Twin-sensor Array based on a Combination of Mach-Zehnder and Michelson Interferometers", Journal of Intelligent Material Systems and Structures, 0: 1045389X08097955v1

[19] NASA (2009). "Nasa Evaluates Fiber Optic Technology for Future Aircraft Efficiency", release 08-31, http://www.nasa. gov/centers/dryden/news/NewsReleases/2008/08-31.html, Extracted on 25 July

[20] Yun JR, Yanbiao L, Gang DP, (2007). "Direct and simultaneous detection of temperature and strain using combined Brillouin and Raman backscatters in single mode fiber" (Proceedings Paper) Advanced Sensor Systems and Applications III, Editors, 683021

[21] Crisp J (2001) "Introduction to Fiber Optics", 2nd Edition, Newnes, An imprint of Butterworth-Heinemann, Linacre House, Jordan Hill, Oxford OX2 8DP, A division of Reed Educational and Professional Publishing Ltd. ISBN 07506 50303

[22] Hill KO, Fujii Y, Johnson DC and Kawasak BS (1978). "Photosensitivity in optical fiber waveguides: application to reflection filter fabrication," Applied Physics Letters, 32, 647-649

[23] Neyer A, Wittmann B and Johnck M (1999). "Plasticoptical-fiber-based parallel optical interconnects", IEEE Journal of Selected Topics in Quantum Electronics, 5(2), 193-200

[24] Koike Y (2008), "Microoptics and Photonics Polymer", Japanese Journal of Applied Physics, 47, 6629-6634.

[25] Kenichi I and Noboru Y (1977), "Plastic focusing fiber for imaging applications," Applied. Optics, 16, 1305-1310

[26] Dandridge A (1991). "Fiber Optic Sensors Based on the Mach-Zehnder and Michelson Interferometers", Fiber Optic Sensors: An Introduction for Engineers and Scientists, Wiley. New York.

[27] Kersey, AD andDandridge A (1990). "Applications of fiber-optic sensors", IEEE Transactions on Components, Hybrids, and Manufacturing Technology, 13(1), 137-143

[28] Peterson JI and Vurek GG (1984). "Fiber-optic sensors for biomedical applications", Science Magazine, 224(4645), 123-127

[29] Vera R, Giancarlo CR, Stefano S and Silvana T (1984). "Lens-ended fibers for medical applications: a new fabrication technique," Applied. Optics, 23, 3277-3283

[30] Krohn DA. ( 2000), "Fiber Optical Sensors, Fundamentals and Applications", 3rd Ed., Research Triangle Park, NC, Instrument Society of America.

[31] Fabin S (2006), "UV-Induced Intrinsic Fabry-Perot Interferometric Fiber Sensors and Their Multiplexing for Quasi-Distributed Temperature and Strain Sensing”, PhD Thesis, Virginia Polytechnic Institute and State University

[32] Li H N, Li D S and Song G B (2004). "Recent applications of fiber optic sensors to health monitoring in civil engineering," Engineering Structures, 26, 1647-1657

[33] Rao YJ and Jackson DA (1996). "Recent progress in fibre optic low-coherence interferometry," Measurement Science Technology, Vol. 7(7), 981-999

[34] Claus RO, Gunther MF, Wang AB, Murphy KA and Sun D (1993). "Extrinsic Fabry-Perot Sensor for Structural Evaluation," Applications of Fiber Optic Sensors in Engineering Mechanics, ASCE, 60-70

[35] Yuan L and Ansari F (1997). "White Light Interferometric Fiber Optic Distributed Strain Sensing System," Sensors and Actuators,Part A, Physical, 63(3), 177-184

[36] Maaskant R, Alavie T and Measures M (1998). "”A Recent Experience in Bridge Strain Monitoring with Fiber Grating Sensors," Fiber Optic Sensors for Construction Materials and Bridges, CRC, 129-135

[37] Vohra ST, Chang CC, Danver BA, Althouse B, Davis MA and Idriss R (1998). "Preliminary Results on the Monitoring of an In-service Bridge Using a 32-Channel Fiber Bragg Grating Sensor System," Fiber Optic Sensors for Construction Materials and Bridges, CRC, 148-158

[38] Spillman W and Lord J (1987). "Self-referencing multiplexing technique for fiber-optic intensity sensors", Journal of Lightwave Technology, 5(7), 865 - 869. Todd MD, Johnson GA and Vohra ST (2001). "Deployment of Fiber Bragg Grating-Based Measurement System in a Structural Health Monitoring Application," Smart Materials and Structures, 2001, 10, 534-539

[39] Casas JR and Cruz PJS (2003). "Fiber Optic Sensors for Bridge Monitoring", Journal of Bridge Engineering, ASCE, $8(6), 362-373$

[40] Meltz G, Morey WW and Glenn WH (1989). "Formation of Bragg gratings in optical fibers by a transverse

[41] Maaskant R, Alaie AT, Measures RM, Tadros G, Rizkalls SH, Guha-Thakurta (1997). "A Fiber-Optic Bragg Grating Sensors for Bridge Monitoring," Cement and Concrete Composites, 19, 21-33

[42] Todd MD, Johnson GA and Vohra ST (2001). "Deployment of Fiber Bragg Grating-Based Measurement System in a Structural Health Monitoring Application," Smart Materials and Structures, 2001, 10, 534-539

[43] Moyo P, Brownjohn JMW, Suresh R and Tjin SC (2005). "Development of Fibre Bragg Grating Sensors for Monitoring Civil Infrastructure,” Engineering Structures, 27, 1828-1834

[44] Guemes, A. (2002). "Optical fiber sensors." "'Preconference course: Health monitoring techniques: An overview." Structural health monitoring, monitoring, Ecole Normale Supe'rieure de Cachan, Paris

[45] Mawatari T and Nelson D (2008) "A multi-parameter Bragg grating fiber optic sensor and triaxial strain measurement", Smart Materials and Structures, 17 (3), 035033

[46] Tennyson R C, Mufti A A, Rizkalla S, Tadros G and Benmokrane B (2001) "Structural health monitoring of innovative bridges in Canada with fiber optic sensors", Smart Materials and Structures, 10 (3), 560, doi: 10.1088/ 0964-1726/10/3/320 
[47] Zhou Z and Ou J (2004). "Development of FBG sensors for Structural Health Monitoring in civil infrastructures," Proceeding of North American Euro-Pacific Workshop, Sensing Issues in Civil Structural Health Monitoring", Waikiki Beach, Oahu ,Hawaii ,USA

[48] Merzbacher CI, Kersey AD and Friebele EJ (1996). "Fiber optic sensors in concrete structures: A review", Smart Materials and Structures, 5(2), 196-208

[49] Guan ZG, Zhang AP, Liao R and He S (2007), "Wavelength Detection of Coherence-Multiplexed Fiber-Optic Sensors Based on Long-Period Grating Pairs", Sensors Journal, IEEE, $7(1), 36-37$

[50] Suresh R, Tjin S C and Ngo N Q (2005) “ Application of a new fiber Bragg grating based shear force sensor for monitoring civil structural components, Smart Materials and Structures, 14, 982-988

[51] Boonsong S, Massood TA and Alex K (1999). "Pd-coated elastooptic fiber optic Bragg grating sensors for multiplexed hydrogen sensing," Sensors and Actuators B: Chemical, 60(1), $27-34$

[52] Golnabi H, Bahar M, Razani M, Abrishami M and Asadpour A (2006). "Design and operation of an evanescent optical fiber sensor," Optics and Lasers in Engineering, 45(1), 12-18

[53] Duym SWR and Lumori MLD (1998). "Distorted laser interferometric angle measurements of a disk drivepivot,"IEEE/ASME Transactions on Mechatronics, 3(4), $265-274$

[54] Trpkovski S, Wade SA, Baxter GW and Collins SF (2003). "Dual temperature and strain sensor using a combined fiber Bragg grating and fluorescence intensity ratio technique in Er3+-doped fiber", Review of Scientific Instruments, 74(5), 2880-2885

[55] Yang Y, Annamdas V G M, and Ferrand H (2007). "Structural health monitoring using smart sensors (Final report on Telokh Blangah Site)". Report for The Entreprise Challenge project collaboration between Sif-U and NTU, submitted to DSTA, Singapore

[56] Cranch GA, Nash PJ and Kirkendall CK (2003). "Large-scale remotely interrogated arrays of fiber-optic interferometric sensors for underwater acoustic applications," Sensors Journal, IEEE, 3(1), 19 - 30

[57] Nakstad H and Kringlebotn J T (2008). "Oil and gas applications: Probing oil fields," Nature Photonics, 2, 147-149

[58] Michael W, Briggs \& Mark W. Farine - Sercel Inc., Houston Texas (2004). "Equipment options in the effort of collecting 4C/4D seismic," Workshop "4D Seismic Applied to the Engineering Reservoir” 22 - 24 June, Rio de Janeiro, Brazil

[59] Borenstein J, Everett HR and Feng L (1996). "Sensors and Methods for Mobile Robot Positioning," University of Michigan

[60] Ghosh SK, Sarkar SK, Chakraborty S and Dan S (2006a). "High frequency electric field effect on plane of polarization in single mode optical fiber," Proceedings, Photonics

[61] Ghosh SK, Sarkar SK and Chakraborty S (2002). "Design and development of a fiber optic intrinsic voltage sensor,"
Proceedings of the 12th IMEKO TC4 international symposium Part 2 (Zagreb, Croatia): 415-419

[62] Ghosh SK, Sarkar SK, Chakraborty S and Dan S (2006b). "A proposal for single mode fiber optic watt measurement scheme," Journal of Optics (Calcutta) (Optical Society of India) 35 (2): 118-124

[63] Zeller $M$ and Scheer G (2008). "Add Trip Security to Arc-Flash Detection for Safety and Reliability," Proceedings of the 35rd Annual Western Protective Relay Conference, Spokane, WA"

[64] Wikipedia (2011).

http://en.wikipedia.org/wiki/Fiber_optic_sensor, Accessed data 31 st July

[65] Grossman BG, Yongphiphatwong T and Sokol M (2005). "In situ device for salinity measurements (chloride detection) of ocean surface”, Optics \& Laser Technology, 37(3), 217-223

[66] Luo F, Liu J, Ma N and Morse T F (1999). "A fiber optic microbend sensor for distributed sensing application in the structural strain monitoring", Sensors and Actuators A: Physical, 75(1), 41-44

[67] Yurek AM (1992). "Real World Applications Of Interferometric Fiber Optic Sensors," Lasers and Electro-Optics Society Annual Meeting, 1992. LEOS apos;92, Conference Proceedings

16-19 Nov, 452 - 453

[68] Deng J (2004). "Development of Novel Optical Fiber Interferometric Sensors with High Sensitivity for Acoustic Emission Detection", PhD Dissertation, Blacksburg, Virginia

[69] Zhang Y (2005). "Miniature fiber-optic multicavity Fabry-Perot interferometric biosensor", PhD Dissertation, Blacksburg, Virginia

[70] Griffin B, Connelly MJ (2004). "Digital signal processing of interferometric fiber optic sensors," Proceedings of the Lightwave Technologies in Instrumentation and Measurement Conference, 19-20 Oct. pages:153 - 156

[71] Kersey AD, Marrone MJ, Dandridge A and Tveten AB (1988). "Optimization and stabilization of visibility in interferometric fiber-optic sensors using input-polarization control," Journal of. Lightwave Technologyl. 6, 1599-1609

[72] Marrone MJ, Kersey AD and Dandridge A (1992). "Fiber optic michelson aft with passive elimination of polarization fading a ource feedback isolation," Optical Fiber Sensors Conference, 8, 69- 72

[73] Chien PY, Chang YS and Chang MW (1996), "Vibration Suppression in a Flexible Structure Based on Fiber Optics Michelson Interferometric Sensor," Journal of Intelligent Material Systems and Structures, 7(1), 65-70

[74] Kondrat M, Szustakowski M, Pałka N, Ciurapiński W and ŻyczkowskiM (2007). "A Sagnac-Michelson fibre optic interferometer: Signal processing for disturbance localization,' Opto-Electronics Review, 15(3), 127-132

[75] Changhai S, Jianping C, Guiling W, Xinwan L, Junhe Z and Fang O (2006). "Stable dynamic detection scheme for magnetostrictive fiber-optic interferometric sensors," Optics Express, 14(12)

[76] Caldas P, Jorge PAS, Araújo FM, Ferreira LA, Manuel B. 
Marques Gaspar Rego and Santos JL (2008). "Fiber modal Michelson interferometers with coherence addressing and heterodyne interrogation," Proceeding of SPIE, Optical Engineering, 47, 044401

[77] Bottino L.J (2003). "Optical communications-the origins of the state of the art",The 22nd Digital Avionics Systems Conference. DASC apos; 03., 2, 6.B.5- 61-13, Oct.12-16

[78] Wickersheim (1991). “Application of Fiber Optic Thermometry to the Monitoring of Winding Temperatures in Medium and large Power Transformers", SPIE Optic laser Sensors IX, 1584, 3-14

[79] Saravolac MP (1994). "The use of optic fibres for temperature monitoring in powertransformers", IEE Colloquium on Condition Monitoring and Remanent Life Assessment in Power Transformers, 7/1 - 7/3, INSPEC Accession Number: 4673523

[80] Betta G, Pietrosanto A, Scaglione A (2001). "An enhanced fiber-optic temperature sensor system for power transformer monitoring", IEEE Transactions on Instrumentation and Measurement, 50(5), $1138-1143$

[81] Sensornet (2009) "Sensornet's Digital Fiber optical sensors", http://www.sensornet.co.uk, accessed on 31st July

[82] Lee LR, Ryu CY, Koo BY, Kang SG, Hong CS and Kim CG (2003). "In-flight health monitoring of a subscale wing using a fiber Bragg grating sensor system," Smart Materials and Structures, 12, 147-155

[83] Science Daily (2007) "Networks of sensors mounted on commercial aircraft", Release of news on July 30

[84] SANDIA (2009) Http://www.sandia.gov

[85] Latini V, Striano V, Coppala G and Rendina I (2007). "Fiber optic sensors system for high-temperature monitoring of aerospace structures," Photonic Materials, Devices, and Applications II, 6593, 65930S

[86] Weedinstrument (2007) "Fiber Optic Self healing Application" released on 26 Oct 2007 , http://www.weedinstrument.com

[87] Read IJ and Foote PD (2001). "Sea and flight trails of optical fibre Bragg grating strain sensing systems," Smart Materials and Structures, 10, 1085-1094

[88] Ohno H, Naruse H, Kihara M and Shimada A (2001). "Industrial Applications of the BOTDR Optical Fiber Strain Sensor", Optical Fiber Technology, 7(1), 45-64

[89] Wang G, Pran K, Sagvolden G, Havsgård BG, Jensen AE, Johnson GA and Vohra ST (2001). "Ship hull structure monitoring using fibre optic sensors", Smart Materials and Structures 10(3), 472-478

[90] Kasahara J, Sato T, Momma H, and Shirasaki Y (1998). “A new approach to geophysical real-time measurements on a deep-sea floor using decommissioned submarine cables", Earth Planets Space, 50, 913-925, 1998

[91] McPhaden MJ (1995). "The tropical atmosphere ocean array is completed", Bulletin of the American Meteorological Society, 76, 739-741

[92] Milburm HB, McLain PD and Meining C (1996). "ATLAS buoy reengineering for next decade", Proceedings of
Ocean '96, 698-702, MTS/IEEE, Florida, U.S.A

[93] Shimura S (1984). “.International Submarine Cable Systems”, KDD Engineering and Consulting, Inc, 507, Tokyo

[94] Morison D and Tennyson R (2007). "Direct Assessment Of Corrosion Using Fiber Optic Sensors”, Corrosion, March 11 15, Paper Number 07385, NACE International

[95] Moxa (2009) "Oil refinery remote monitoring and control system under hazardous environments"

,http://www.moxa.com/applications/success_stories_Oil_Ref inery_Remote_Monitoring_and_Control.htm, accessed on 31 st July

[96] Kersey AD (2000). "Optical fiber sensors for permanent down well monitoring applications in the oil and gas industry," IEICE Transactions on Electron E83-C(3), 400-404

[97] Kersey AD, Davis MA and Patrick HJ (1997). "Fiber Grating Sensor," Journal of Lightwave Technology, 15, 1442-1461

[98] Brower DV and Prescott N (2004). " Real time subsea monitoring and control smart field solutions", June 3, Subsea Rio Conference

[99] Chong KP, Carino NJ and Washer G (2003). "Health monitoring of civil infrastructure," Smart Materials and Structures, 12, 483-493

[100] Zhou Z and Ou J (2004). "Development of FBG sensors for Structural Health Monitoring in civil infrastructures," Proceeding of North American Euro-Pacific Workshop, Sensing Issues in Civil Structural Health Monitoring", Waikiki Beach, Oahu ,Hawaii ,USA

[101] Merzbacher CI, Kersey AD and Friebele EJ (1996). "Fiber optic sensors in concrete structures: A review", Smart Materials and Structures, 5(2), 196-208

[102] Leng JS, Barnes RA, Hameed A, Winter D, Tetlow J, Mays GC and Fernando GF (2006). "Structural NDE of concrete structures using protected EFPI and FBG sensors", Sensors and Actuators A: Physical, 126 (2), 340-347

[103] Fuhr P L and Spammer S (1998). "Fiber optic sensors in the Waterbury bridge," Proceedings of SPIE, 3489, 124-129

[104] Hong N. L, Dong S. L and Gang B. S (2004). "Recent applications of fiber optic sensors to health monitoring in civil engineering," Engineering Structures, 26, 1647-1657

[105] Healey BG, Matson RS and Walt DR (1997). "Fiberoptic DNA Sensor Array Capable of Detecting Point Mutations", Analytical Biochemistry, 251(2), 270-279

[106] Sutapun B, Azar MT and Kazemi A (1999). "Pd-coated elastooptic fiber optic Bragg grating sensors for multiplexed hydrogen sensing", Sensors and Actuators B: Chemical, 60(2), 27-34

[107] Mignani AG, Falciai R and Trono C. (2000). "Optical Fiber Sensors for the Cultural Heritage", Optical Sensors and Microsystems: New Concepts Materials Technologies, Kluwer Academic / Plenum Publishers, New York. 2000, 978-0-306-47099-8

[108] Wolfbeis OS (2006). "Fiber-Optic Chemical Sensors and Biosensors", Analytical Chemistry, American Chemical Society, 78 (12), 3859-3874

[109] Mignani AGand Baldini F (1995). “In-vivo biomedical 
monitoring by fiber-optic systems", Journal of Lightwave Technology, 13(7), 1396-1406

[110] Spillman JWB, Mayer M, Bennett J, Gong J, Meissner KE, Davis B, Claus RO, Muelenaer JAA and Xu X (2004). "A 'smart' bed for non-intrusive monitoring of patient physiological factors", Measurement Science and Technology, 15(8), 1614-1620

[111] Bluestein BI, Walczak IM, Chen SY (1990). "Fiber optic evanescent wave immune sensors for medical diagnostics", Trends Biotechnology,8(6):161-8

[112] Katzir A (1993), Editor. "Optical Fibers in Medicine VIII". Proceedings of SPIE, 1893

[113] Lieberman RA (1993). "Chemical, Biochemical, and Environmental Fiber Sensors V”, Proceedings of SPIE

[114] Milanovich FP (1993). Editor. "Fiber Optic Sensors in Medical Diagnostics", Proceedings of SPIE, 1886

[115] Inaudi D (2001). “Application of optical fiber sensor in civil structural monitoring," Proceedings of SPIE 4328, 1-10 (2001)

[116] Petrušis A, Rector J, Smith K, de Man S, and Iannuzzi D (2009). "Align-and-shine photolithography: batch production of photolithography patterns on the tip of a fiber", Journal of Micromechanics Microengineering, 19(4), 047001

[117] Xia H, Mu H, Yang Y and Sun D (2007). "Direct and simultaneous detection of temperature and strain using combined Brillouin and Raman backscatters in single mode fiber", Advanced Sensor Systems and Applications III, 683021

[118] Zou L, Bao X, Yang S, Chen L, and Ravet F (2006a). "Effect of Brillouin slow light on distributed Brillouin fiber sensors", Optics Letters, 31, 2698-2700

[119] Zou L, Bao X, Ravet F and Chen L (2006b). "Distributed Brillouin fiber sensor for detecting pipeline buckling in an energy pipe under internal pressure," Applied Optics, 45, $3372-3377$

[120] Zou L, Ferrier G, Afshar S, Yu Q, Chen L, and Bao X, (2004a). "Distributed Brillouin scattering sensor for discrimination of wall-thinning defects in steel pipe under internal pressure," Applied Optics, 43, 1583-1588

[121]Zou L, Bao X, Wan Y and Chen L (2005). "Coherent probe-pump-based Brillouin sensor for centimeter-crack detection," Optics Letters, 30, 370-372

[122] Zou L and. Feng MQ (2008). "Detection of micrometer crack by Brillouin-scattering-based distributed strain and temperature sensor," 19th International Conference on Optical Fiber Sensors, Perth (Australia, 14-18 April)

[123] Zou L, Bao X, Afshar S and Chen L (2004b). "Dependence of the Brillouin frequency shift on strain and temperature in a photonic crystal fiber”, Optics Letters, 29, 1485-1487

[124] Xie J and Pecht M (2004). “Applications of In-situ Health Monitoring and Prognostic Sensors, The 9th Pan Pacific Microelectronics Symposium Exhibits \& Conference, Kahuku, Oahu, Hawaii, 10-12 February

[125] Inaudi D (2007). "Fiber Optic Sensors for Structural Monitoring", Proceedings of the OPTIMESS Workshop, 28th-30th, May, Leuven, Belgium

[126] Udd E (1994). Editor, "Fly-by-Light", Proceedings of SPIE, 2295

[127] Morey WW, Meltz G and Glenn WH (1989). “Bragg-Grating Temperature and Strain Sensors", Proceedings of Optical Fiber Sensors 89, p. 526, Springer-Verlag, Berlin

[128] Ball GA, Meltz G and Morey W W (1993). "Polarimetric Heterodyning Bragg-Grating Fiber Laser", Optics Letters, 18: 1976, 1993

[129] Wolfbeis OS and Greguss P (1993). "Biochemical and Medical Sensors", Proceedings of SPIE, 2085 